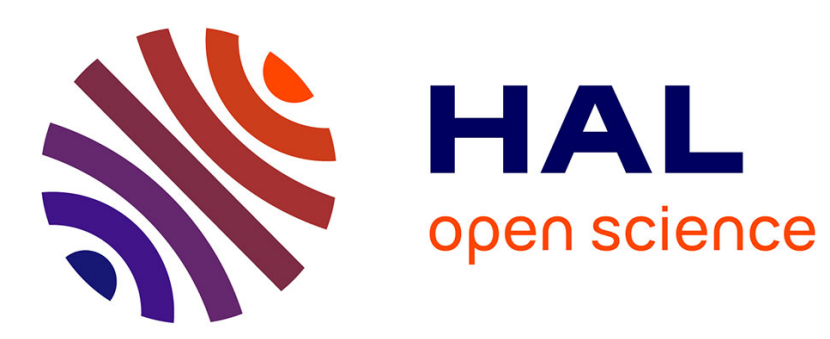

\title{
Negative Differential Resistance, Oscillations and Constrictions of Low Pressure, Low Current Discharges
}

Z. Petrovic, I. Stefanovic, S. Vrhovac, J. Zivkovic

\section{To cite this version:}

Z. Petrovic, I. Stefanovic, S. Vrhovac, J. Zivkovic. Negative Differential Resistance, Oscillations and Constrictions of Low Pressure, Low Current Discharges. Journal de Physique IV Proceedings, 1997, 07 (C4), pp.C4-341-C4-352. 10.1051/jp4:1997428 . jpa-00255584

\section{HAL Id: jpa-00255584 https://hal.science/jpa-00255584}

Submitted on 1 Jan 1997

HAL is a multi-disciplinary open access archive for the deposit and dissemination of scientific research documents, whether they are published or not. The documents may come from teaching and research institutions in France or abroad, or from public or private research centers.
L'archive ouverte pluridisciplinaire HAL, est destinée au dépôt et à la diffusion de documents scientifiques de niveau recherche, publiés ou non, émanant des établissements d'enseignement et de recherche français ou étrangers, des laboratoires publics ou privés. 


\title{
Negative Differential Resistance, Oscillations and Constrictions of Low Pressure, Low Current Discharges
}

\author{
Z.Li. Petrović, I. Stefanović, S. Vrhovac and J. Živković \\ Institute of Physics, University of Belgrade, P.O. Box 57, 11001 Belgrade, Yugoslavia
}

\begin{abstract}
A brief review is given of the recent experimental and theoretical studies of low current diffuse discharges. Two models are developed, one that is based on phenomenological description by effective discharge circuit parameters and the other which is based on the calculation of the field profile from the ion distribution for uniform field. In the first case the physical process responsible for the development of the negative differential resistance is the dependence of the secondary electron yield on current through mudification of the field close to the cathode. Experimental systems were developed to provide observables that include: breakdown voltage, voltampere characteristics (which in the low current limit is represented very well by a negative differential resistance), limits and the profile of the low current oscillations, frequency and damping of the induced oscillations, current growth coefficient and the onsets for constrictions. All of the observables are very well predicted by the theory based on the data taken from independent sources, once the steady state secondary electron yield has been fitted to predict the breakdown voltage.
\end{abstract}

\section{INTRODUCTION}

In this paper we describe the results of studies of low-pressure, low-current but very high $E / N$ discharges operating in the low current diffuse (Townsend dark) regime and of their transitions to constricted (normal) glow discharge. The experimental procedure and models were initially developed at the University of Colorado by Phelps and coworkers [1-4] and were further developed at the University of Belgrade $[5,6]$. The discharges studied here are obstructed or cathode dominated and are a good model for cathode fall or for some of the processes occurring in If discharge sheaths.

The primary motive for these studies came from experimental observations that these discharges are inherently unstable (oscillate) even at very low currents, below the transition to constriction. Thus since we could not beat (remove) the oscillations we have joined them (started studying). While some evidence of negative differential resistance, which could lead to self sustained oscillations, existed in the literature [7-9] we believed that the effect so far has not been fully understood and generally appreciated.

\section{EXPERIMENTAL SETUP AND PROCEDURE}

The discharge vessel (see Figure 1a) consists of parallel plane electrodes placed inside a quartz cylinder that prevents the long path breakdown. Thus we are able to operate both to the left and to the right of the Paschen minimum. The parallel electrodes can be positioned at several fixed distances ranging between 1 and $3 \mathrm{~cm}$, but it is necessary to open the system to adjust the gap. In order to provide parallel surfaces, one electrode is fixed to a dielectric supports with different thicknesses. The test whether the surfaces are sufficiently parallel is performed by observing the radial distribution of emission in the diffuse low current regime (Townsend regime) and making sure that the discharge is not confined to one region only. The diameter of the electrodes in both experiments was of the order of $8 \mathrm{~cm}\left(\operatorname{area}=50 \mathrm{~cm}^{2}\right)$ while the gap was $1.05 \mathrm{~cm}$. In the variable gap measurements, gaps of 1,2 and $3 \mathrm{~cm}$ were used. 


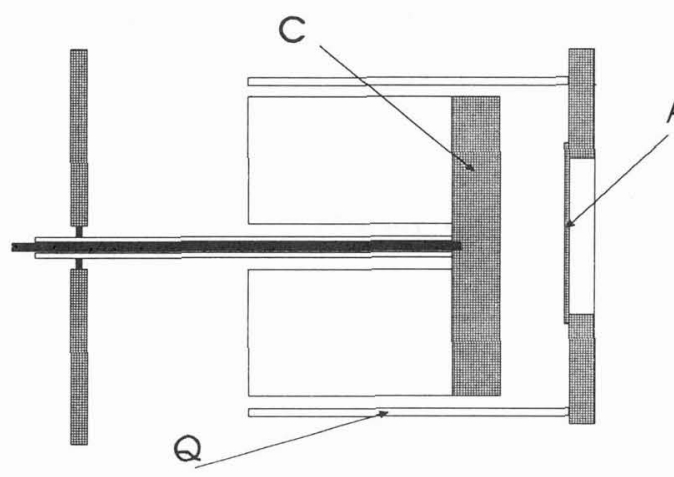

a)

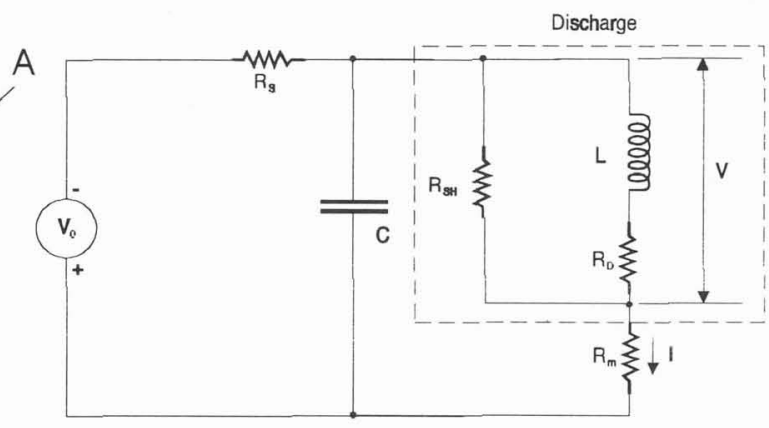

b)

Figure 1: a) Discharge vessel: C-cathode, A-anode, Q-quartz tube and b) equivalent electrical circuit of the discharge.

The electrical circuit of the experiment is shown in Figure 1b. The system has provision for producing a pulse of current (up to $20 \mathrm{~mA}$ ) in addition to a very small dc current (3-10 $\mu \mathrm{A}$ ). The dc current is required to remove the breakdown time delays and achieve reproducible operating conditions. The dc current is as small as possible in order to avoid heating and/or significant conditioning of the cathode during the measurements. Yet it is not possible to maintain a stable dc current under all conditions (pressure, gap, gas).

Initial mode of operation was to use laser induced perturbation [1]. Discharge was operated at a low dc current. A pulse of a $\mathrm{Q}$ switched YAG laser, quadrupled in frequency, lasting 10-15 ns was focused onto the cathode inducing secondary electrons from the cathode and producing a pulse of current. This mode did not provide a possibility to record voltage current characteristic over a wide range of currents. It also required averaging over several shots to produce a smooth signal with a possible uncertainty due to the jitter. Yet laser pulsing provided data for ringing frequencies and damping corresponding exactly to the dc operating conditions, i.e. without questions whether the discharge has fully relaxed which may be relevant to the voltage pulsing technique. The voltage pulsing, on the other hand, is more versatile [2,5] and easier to perform. All the data presented here were obtained by voltage pulsing.

Before measurements, the surfaces of electrodes were treated by a relatively high current of the order of few $\mathrm{mA}$ until a stable breakdown voltage $V_{\mathrm{b}}$ was achieved. Initially, gold plated cathodes were used. We found that it is possible to achieve stable and reproducible operating conditions after treating the cathode to a hydrogen or argon discharge. Gold plated cathodes gave rise to quite large variations of operating conditions during the treatment, with breakdown voltages changing by a factor even greater than two. Gold plated electrodes had to be treated every day. Using a copper cathode, without gold, gives smaller variations of the breakdown voltage and it was possible to achieve reproducible conditions over periods of several weeks. The behavior of the two types of electrodes is consistent with formation of a very thin film of yet unknown nature on gold which is removed by ion bombardment. On the other hand the copper has a stable oxide layer which determines its secondary electron yield. If not treated, gold plated cathode will significantly change its properties during the measurements. In case of the copper cathode, even a treatment of the surface by currents of few $\mathrm{mA}$ over periods of up to one hour is not sufficient to remove the oxide film. The presence of a thin oxide layer may give stable operating conditions but may make it difficult to apply secondary electron yield measurements from an independent experiment due to a possible Malter effect.

We usually use a monitoring resistor $R_{\mathrm{m}}$ to determine the current in the low voltage circuit between the anode and the ground, but it is possible to run the discharge with zero resistance and still obtain the value of the current by applying a low input impedance amplifier. After a stable, low dc current, operation is achieved, pulses of higher current are triggered lasting only sufficiently long to make a reliable recording of voltage and current. Voltage is measured by two probes, as a difference, when a relatively 
high monitoring resistor is used in the low voltage anode circuit to determine the current. Probes are connected directly to the discharge electrode terminals and remain connected even when not used. The effective capacitance of the electrode system and of the terminals is measured by pulsing the system without the discharge operating. The voltage and current transients are recorded by a digital oscilloscope and stored in a computer. In Figure 2 we show an example of voltage and current pulses recorded by the technique. It should be noted that even though the external voltage pulse is positive, the voltage change at the discharge is negative indicating a negative differential resistance.

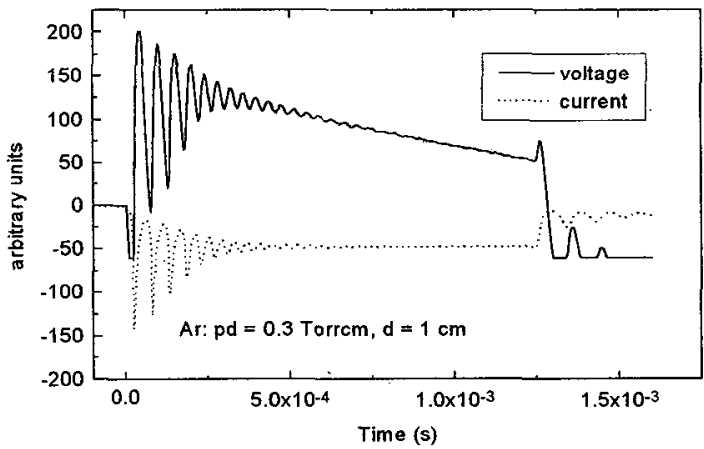

Figure 2: Voltage and current waveforms. The sloping voltage pulse is due to the discharging of capacitor, extrapolation to zero time is used to establish the voltage drop.

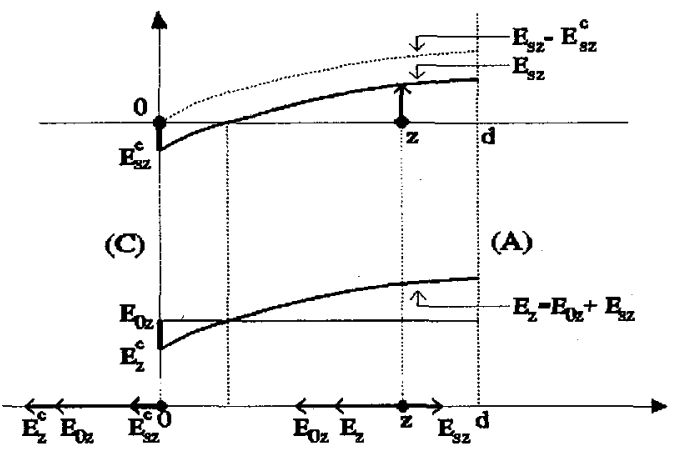

Figure 3: Electric field distribution in the gap between electrodes. Space charge effect (s) is integrated and added to the homogeneous field. Field at the cathode is denoted by the superscript ' $c$ '.

Recorded waveforms are fitted to obtain voltage and current before and after the pulse. The signal in the transient region is used to obtain the frequency and damping of damped oscillations induced by the high current pulse. When transition is made to the selfsustained oscillations the duration of the pulse is extended as much as possible to verify the regime and the waveform is recorded to give the frequency and for comparisons with calculations. The onset of selfsustained oscillations is recorded both by using a pulsed technique and by using dc measurements. Another observable that is recorded is the rate of increase of current when the pulse is applied $[3,4]$. It is recorded over sufficiently short time so it is possible to assume that it corresponds to a linear increase in voltage. In order to avoid a possible effect of the voltage pulse on detection of current, a differential amplifier is used with compensation adjusted for pulses when no discharge is created.

One of the electrodes can be made transparent by depositing a thin metallic film on glass. Such electrodes are used to study constrictions with a CCD camera that was needed to record the spatial distribution of light. This method gave only a limited possibility of getting a time resolved results so a photomultiplier was used to record the emission through the electrode as well.

So, to summarize, the observables in the experiment are: breakdown voltage, voltage-current characteristics and consequently the negative differential resistance, range of oscillations (damped and self sustained), frequency and damping of damped oscillations and the rate of rise of current. It is also possible to record the onset of constrictions which also corresponds to the behavior of the discharge in the low current regime.

\section{MODELS}

The basis for the model developed to describe the phenomena in low-pressure, low-current cathode dominated diffuse discharges is the idea that under those conditions a gradual accumulation of the spacecharge effects affects the uniformity of the electric field. The field increases close to the cathode due to the shielding by charged particles and decreases elsewhere (see Figure 3). An increase in the field in a small region leads to a drastic increase of electron production due to the $E / N$ dependence of the ionization 
coefficient. Thus the decrease in other regions may be greater and the overall voltage decreases giving rise to a negative differential resistance. The perturbation of the field is assumed to be small so the initial distribution of charges used for calculation of the space charge effects is that for the unperturbed field. Another assumption is that of locality. It has two aspects. One is that we can represent the ionization rate coefficient by its local field value, which may be questionable for very high $E / N$ conditions. Yet it was shown by Stojanović and Petrović [10] that even when electron beams develop it is still possible to assign a reasonably well defined value to the ionization coefficient up to very high $E / N$, much higher than the upper limit covered here. A more important aspect of locality is that the secondary electron yield at the cathode is determined by the field right at the cathode, implying equilibrium for ions. This assumption may fail for large mean free paths.

Two different models were developed to analyze the experimental data [3]. The first is a phenomenological model which basically means that some of the physical phenomena were assigned to effective circuit parameters of the discharge. The second model is more physical as it attempts to correlate observables with physical parameters such as ionization and secondary electron yield coefficients and their dependence on $E / N$.

\subsection{Phenomenological Model}

The basis for the phenomenological model is an idea that the secondary electron yield may be represented as a function of both voltage and current:

$$
\gamma=\gamma_{p}+k_{V} V+k_{I} I
$$

The first assumption is easily understood, while the second requires further explanation. The current dependence of gamma is due to the fact that increasing of the current leads to an increased space charge shielding effect and consequently a higher field close to the electrode (while overall voltage may drop). Thus in principle both dependencies are associated with the field close to the cathode.

Another assumption of the model is that the changes occur on the time scales greater than the ion transit time $T$. Thus it is possible to associate the electron current $I_{e}$ at the cathode (at time $t+T$ ) with the current at the time $t$ by equation:

$$
I_{e}(0, t+T)=I_{n}(0, t+T)+\mathscr{H}_{e}(0, t)[\exp (\alpha(t) d)-1]+\delta I_{e}(0, t) \exp (\alpha(t) d),
$$

where $I_{p}(0, t+T)$ is the photoelectric current produced by irradiation of the cathode and $\delta$ is the secondary electron production term due to impact of fast electrons on anode surface.

After applying Taylor expansion and using definitions of coefficients this equation may be converted to:

$$
\frac{d I}{d t}=\left[\frac{(1+\gamma) I_{p}}{\gamma T}+\frac{(g-1) I}{T}-\frac{I k_{V}}{\gamma(1+\gamma)} \frac{d V}{d t}\right]\left[1+\frac{I k_{I}}{\gamma(1+\gamma)}\right]^{-1},
$$

where $g$ is the round trip charge multiplication factor that may be associated with the breakdown condition:

$$
g(t)=\gamma[(1+\delta) \exp (\alpha(t) d)-1]
$$

The electric circuit of the discharge with effective representation of the discharge is shown in Fig. $1 \mathrm{~b}$ and when we solve the equations for that circuit, another differential equation is obtained:

$$
\frac{d V(t)}{d t}=\frac{1}{R_{S} C}\left[V_{0}(t)-V(t)-I(t)\left(R_{s}+R_{m}\right)\right]-R_{m} \frac{d I(t)}{d t} .
$$

The circuit parameters correspond to those defined in Fig. $1 \mathrm{~b}$.

Equations (3) and (5) are a complete set of equations which may be solved numerically for any signal provided that initial assumptions are acceptable. Analytical solutions may be obtained for a small 
signal theory using the ac component as a small perturbation on the steady state (SS) current $\left(I=I_{s s}+\beta i ; V=V_{s s}+\beta v\right)$. The corresponding equations are:

$$
\begin{gathered}
{\left[1+\frac{I_{s s} k_{I}}{\gamma_{s s}\left(1+\gamma_{s s}\right)}\right] \frac{d i}{d t}=\frac{I_{s S}}{T} \frac{\partial g}{\partial V} v-\frac{I_{s s} k_{v}}{\gamma(1+\gamma)} \frac{d v}{d t}+\frac{I_{s S} k_{I}}{T \gamma_{s s}} i,} \\
\frac{d v}{d t}=-\frac{v}{R_{s} C}-\frac{i}{C}\left[1+\frac{R_{m}}{R_{s}}\right]-R_{m} \frac{d i}{d t},
\end{gathered}
$$

which give formulae for the frequency and damping of induced oscillations:

limits of selfsustained oscillations:

$$
\begin{gathered}
\omega^{2}=\frac{I_{s S}}{R_{S} C T}\left[\left(R_{s}+R_{m}\right) \frac{\partial g}{\partial V}-\frac{k_{I}}{\gamma_{s S}}\right] \times\left[1+\frac{I_{s s}\left(k_{I}-R_{m} k_{V}\right)}{\gamma_{s s}\left(1+\gamma_{s s}\right)}\right]^{-1}-k^{2}, \\
k=\frac{1}{2 R_{S} C}\left[1+\frac{I_{s s}\left(k_{I}-\left(R_{s}+R_{m}\right) k_{V}\right)}{\gamma_{s s}\left(1+\gamma_{S S}\right)}-\frac{R_{S} C I_{S S}}{T}\left(\frac{k_{I}}{\gamma_{S s}}-R_{m} \frac{\partial g}{\partial V}\right)\right], \\
\times\left[1+\frac{I_{S S}\left(k_{I}-R_{m} k_{V}\right)}{\gamma_{s s}\left(1+\gamma_{s S}\right)}\right]^{-1}
\end{gathered}
$$

$$
I_{s s}^{t r}=\left[\frac{R_{s} C}{T}\left(\frac{k_{1}}{\gamma_{s s}}-R_{m} \frac{\partial g}{\partial V}\right)+\frac{\left(R_{s}+R_{m}\right) k_{V}-k_{I}}{\gamma_{s s}\left(1+\gamma_{s s}\right)}\right]^{-1},
$$

and most importantly clearly indicate that the primary cause for the effect of negative differential resistance is the dependence of the secondary electron yield on current $\left(k_{I}\right)$ :

$$
R_{D}=\frac{-k_{I}}{\partial g / \partial V}\left[1+\frac{I_{s s} k_{I}}{\gamma_{s s}\left(1+\gamma_{s s}\right)}\right] \times\left[1+\frac{I_{s s} k_{I}}{\gamma_{s s}\left(1+\gamma_{s s}\right)}\left[1-\frac{k_{V}}{\gamma_{s s} / \partial g}\right]\right]^{-1}
$$

These equations also reveal the influence of external circuit elements on experimental observables.

In the low current limit these equations become:

$$
\begin{aligned}
& \omega^{2}=\frac{1}{L C}\left[1+\frac{\left(R_{m}+R_{D}\right)}{R_{S}}\right], \\
& k=\frac{1}{2 R_{S} C}+\frac{\left(R_{m}+R_{D}\right)}{2 L}, \\
& R_{D}=-\frac{k_{I}}{\gamma_{S S} / \partial V} .
\end{aligned}
$$

\subsection{Physical Model}

It is more physical to represent the secondary yield dependence on the local electric field in front of the cathode rather than on voltage and current. In order to do so one should calculate the field distribution and the shift of the field at the cathode $\left(E_{S}^{C}\right)$ as well as the overall field distribution and the corresponding voltage change $(\delta V)$. This is the basis of the "physical model" of Phelps and coworkers [3]: 


$$
\delta V:=-\int_{0}^{d} E_{s z}(z) d z=-\frac{\hat{\gamma}}{\hat{\gamma}+\gamma \alpha \hat{\alpha} d \exp (\alpha d)} \frac{1}{\varepsilon_{0}} \frac{j}{W_{+}} f^{(1)}\left(\alpha_{0}, d\right),
$$

where

$$
f^{(1)}\left(\alpha_{0}, d\right)=d^{2}\left[\frac{1}{2}+\frac{\exp \left(-\alpha_{0} d\right)}{\alpha_{0} d}-\frac{\left(1-\exp \left(-\alpha_{0} d\right)\right)}{\left(\alpha_{0} d\right)^{2}}\right] .
$$

Implicit assumption of the model is that the ion distribution is that which corresponds to the unperturbed field, which is used in the Poisson equation to determine the field distribution:

$$
\frac{d E_{s z}}{d z}=\frac{1}{\varepsilon_{0}} \frac{j_{z}}{W_{+z}}\left[1-\exp \left(\alpha_{0}(z-d)\right)\right]
$$

When linear terms are retained in Eq. (17) and both electron ionization and secondary yield coefficients are developed to the first term in the electric field (the latter only depends on the field at the cathode), one may obtain an explicit formula for the negative differential resistance normalized to the area $(A)$ of electrodes and the square of the interelectrode spacing $(d)$ :

$$
R_{N}:=\frac{A \delta V}{I d^{2}}
$$

The correlation with the current dependence of the secondary electron yield may also be found:

$$
k_{I}=\frac{d^{2} f(\alpha d) \hat{\gamma}}{A V_{0} \varepsilon_{0} W_{+}}=\frac{d f(\alpha d)}{A \varepsilon_{0} W_{+}} \frac{d \gamma}{d|E|} .
$$

At this point it is important to explain the relationship between the two models. Both models are certainly less accurate than numerical solutions to partial differential equations such as those used in fluid models of if discharges. The two models described here, however, allow us to establish analytical relationships between different quantities and thus to provide physical insight into the main mechanisms. The phenomenological model gives differential equations for voltage and current waveforms which are used to fit the experimantal waveforms and to determine the observables in the experiment. The physical model associates some of the measured quantities with the more fundamental properties of basic physical quantities such as dependence of the secondary electron yield on the electric field. It is not possible to maintain the same level of simplicity of analytical formulae if one attempts to include the electric circuit equations at the level of physical model. In addition the phenomenological model covers a broader range of physical quantities. Therefore it is necessary to maintain application of both models, the first in the analysis of raw experimental data and the second to study the basic causes of the negative differential resistance.

A similar but somewhat modified theory was developed for higher pressures where ions are not the only or the dominant feedback mechanism [11]. Under those conditions photons or metastables may provide a significant effect. For the present, ion dominated, conditions the effect of the modification of the field due to a small but still significant space charge effect is well represented by the formulae above. Calculations of the field profile and deviations from the uniform field distributions of electron and electron density show very small effects for all but the most severe conditions at the onset of transition to the glow (constriction) $[3,4]$.

The present theory is similar, but less restrictive, to the theory of Naumov and Melekhin which was developed earlier and independently of our work, and which has been applied to a more narrow range of experimental data $[12,13]$.

In the remaining of the text we shall compare the theoretical predictions with experimental results. The theory has only one fitting parameter, that is the value of the steady state secondary electron yield which is obtained by fitting the breakdown voltage. It is very strongly affected by surface conditions and therefore has to be obtained directly from the data. All other parameters (in case of hydrogen) are taken from independent sources, mostly experimental. 


\section{U-I CHARACTERISTICS}

The textbooks usually represent volt-ampere characteristics of Townsend dark discharges as a constant with a sudden (usually shown by dashed lines) transition to the constricted glow. The first point is that the negative differential resistance (NDR) i.e. negatively sloped characteristics is usually not discussed, sometimes even not indicated on the graph, even though it has been in the literature since the forties [8]. In fact it appears that a number of specialists in the field are not aware of the universality of the NDR in the dark Townsend regime.

Another point worth discussing is the smooth, uniquely defined appearance of the volt- ampere characteristics as usually presented in the textbooks. Phelps has compiled data for several gases [14] and there are very few examples that the data from different sources agree on the most important properties. In particular the current defining the transition to the constricted regime varies by several orders of magnitude and so does the shape of the dependence. One may, however, accept as expected, the variation of the actual value of the dc breakdown voltage, which is very sensitive to surface conditions.

We have performed a series of measurements for different gases under conditions that were more controlled that those for most other experiments reported in the literature. An essential item was to carefully control that the oscillations did not develop. Another assumed explanation for the large scatter of experimental data in the literature was that local heating of surfaces could cause inconsistencies between different experiments. However, we could not find any evidence of significant heating for currents up to $30 \mathrm{~mA}$ and for the configuration of our experiment [1] even when a thermocouple was positioned within one millimeter from the surface. We however discovered that discharge at moderate and higher currents could modify significantly the conditions at the surface and we had to develop a pulsed technique to limit such effects, which limited possible heating of the surface even further.

\subsection{Data for U-I characteristics}

Gases that were studied include $\mathrm{Ar}, \mathrm{N}_{2}, \mathrm{H}_{2}, \mathrm{CH}_{4}$ and $\mathrm{SF}_{6}$. Thus we cover both atomic and molecular gases, with and without long lived metastables and we also have a representative of electronegative gases. It is also important to note that identity of ions may change for some gases depending on conditions, particular examples are $\mathrm{H}_{2}$ and $\mathrm{SF}_{6}$.

In Figure 4. we show one example of a recorded volt ampere characteristics. As it is difficult to present small changes in voltage on top of a large breakdown voltage, we only present the difference. In Figure 5 voltage difference is shown as a function of $i d^{2}$, which is expected scaling and the agreement between the two characteristics for a factor of three different gap lengths is excellent. The line corresponding to the unscaled negative differential resistance (i.e. as a function of $i$ shown on $i d^{2}$ ) of the discharge is also shown. Most other characteristics for the low current regime for all gases agree very well. The differences in the glow region are however greater.

It is therefore, possible to determine accurately and with great reproducibility volt- ampere characteristics of gas discharges. The profiles agree well with the expected behavior. Through most of the low-current regime it is possible to define a constant negative differential resistance.

\subsection{Negative differential resistance}

Measurements of volt- ampere characteristic show that it is possible to define a constant negative differential resistance (NDR) that describes well most of the characteristics, certainly its low current limit. The second model described here predicts $d^{2}$ scaling for NDR and in Figure 6a we show experimental confirmation of that prediction in case of argon. The case of argon is particularly important as it has only one species of ions. For hydrogen the linearity starts to break down but only slightly at the highest $d$ covered here, indicating a change in the identity of dominant ions as the pressure decreases. For SF 6 (see Figure $6 \mathrm{~b})$ there are two groups of lines in the $R_{\mathrm{D}}$ versus $d^{2}$ graph. The low $p d$ data agree and so do the high $p d$ data, but the two corresponding lines differ. It appears that here, the identity of ions that carry the 
feedback changes as the $p d$ changes. In cases where relevant additional data are available it is possible to predict the values of NDR relatively accurately.

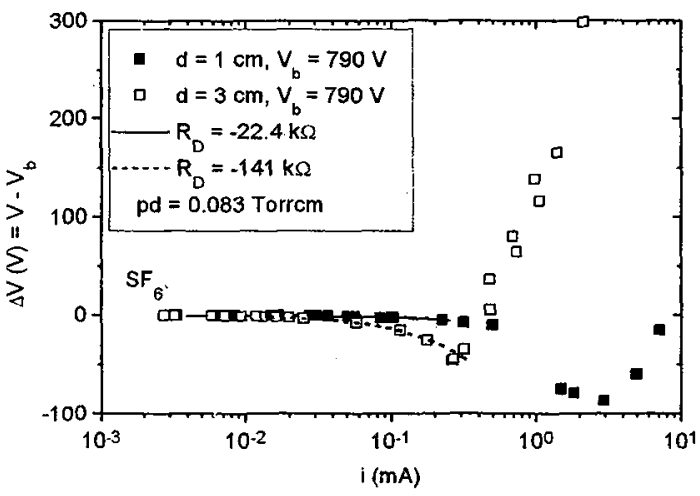

Figure 4: Volt-ampere characteristics for $\mathrm{SF}_{6}$ : symbolsexperimental data for different $d$, lines-fits for $R_{\mathrm{D}}$ [5].

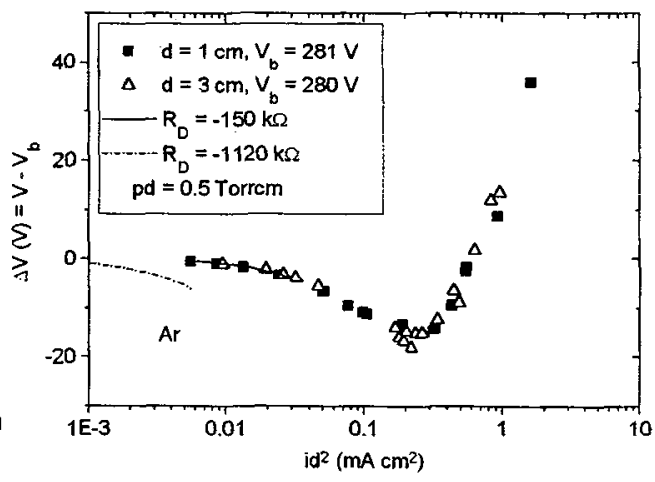

Figure 5: Voltage difference versus id ${ }^{2}$ for Ar: symbolsexperimental data for different $d$, lines-fits for $R_{\mathrm{D}}[5]$.

We have found some situations where NDR starts increasing with a tendency to become infinite close to the onset of constriction. It is believed that this phenomenon is more universal than observed but that oscillations develop once it occurs preventing us from detecting it. The observation has, however prompted the development of a nonlinear theory.

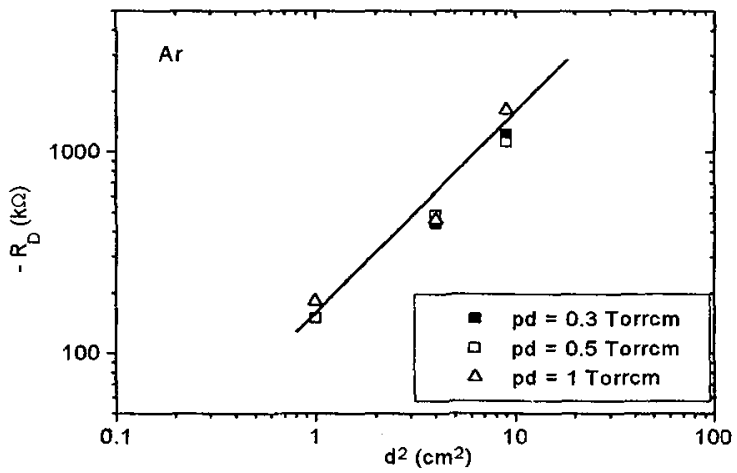

a)

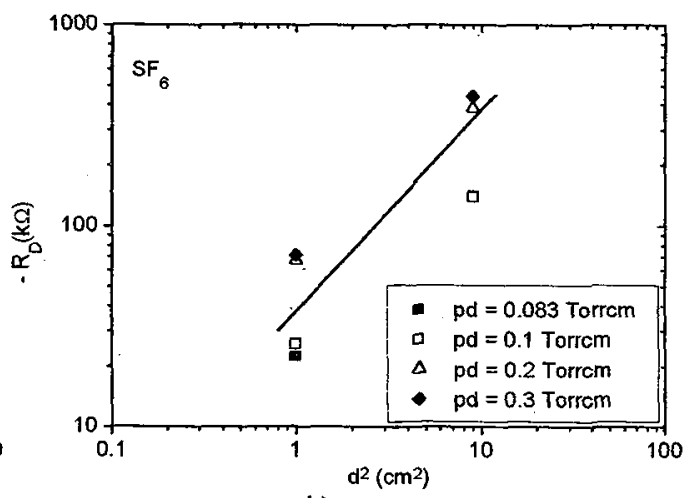

b)

Figure 6: $R_{\mathrm{D}}$ versus $d^{2}$ for $\mathrm{Ar}(\mathrm{a})$ and $\mathrm{SF}_{6}(\mathrm{~b})$ : symbols-experimental data for different values of $p d$ [5]. Solid line indicates a linear dependence.

\subsection{Nonlinear behavior}

Extending development of ionization and secondary yield coefficients beyond the linear term, with a similar extension in the corresponding equations leads to:

$$
\delta V \approx \delta V_{s}^{(1)}+\left(\frac{j_{z}}{\varepsilon_{0} W_{+2}}\right)^{2}\left[\frac{k_{6}}{k_{2}} f^{(2)}\left(\alpha_{0}, d\right)+\left(\frac{k_{3}^{2}}{4 k_{1} k_{2}}-\frac{k_{s}}{k_{2}}\right)\left(f^{(2)}\left(\alpha_{0}, d\right)\right)^{2}\right]
$$

where $\delta V_{s}^{(1)}$ is given by Eq. (15) and

$$
f^{(2)}\left(\alpha_{0}, d\right)=\frac{1}{\alpha_{0}^{2}}\left[d \exp \left(-2 \alpha_{0} d\right)+\alpha_{0} \exp \left(-\alpha_{0} d\right) d^{2}+\frac{1}{3} \alpha_{0}^{2} d^{3}-2 d+\frac{2}{\alpha_{0}}\left(1-\exp \left(-\alpha_{0} d\right)\right)^{2}+\frac{1}{2 \alpha_{0}}\left(1-\exp \left(-2 \alpha_{0} d\right)\right)\right],
$$




$$
\begin{gathered}
k_{6}=-d^{2} \gamma_{0}^{2}\left(\exp \left(\alpha_{0} d\right)\right) \alpha^{\prime \prime}, \\
k_{2}=2 d^{2} \gamma_{0}^{2}\left(\exp \left(\alpha_{0} d\right)\right) \alpha^{\prime}+2 d \gamma^{\prime} .
\end{gathered}
$$

We do not have enough room here to explicitely define the remaining terms in equation (20), a more detailed explanation is given as a contributed paper to this conference [15]. The terms presented here will dominate in case that $f^{(2)}$ is small. The two terms presented here are given explicitely to point out that non-linearities depend directly on derivatives of ionization coefficient and secondary electron yield.

In principle it is also possible to expect non-linearities due to modification of the spatial distribution of ions away from that for the uniform field, due to non-equilibrium effects and also due to emission of resonant photons or metastable diffusion to the surface.

In all measurements, the volt-ampere characteristics is linear in the low current limit, linearity extending quite close to the onset of free nunning oscillations or transition to constrictions. Nevertheless there are few examples of nonlinear behavior away from that transition resulting in a strong current dependence of the negative differential resistance (NDR) [16]. It appears that NDR would have a tendency to extend to very high values right at the transition. It is however not certain yet what the actual cause for the non-constant NDR is, for those examples.

The ultimate non-linearity of course, occurs at the transition to constriction. The point of transition appears to be very accurately defined and there is also a well defined hysteresis.

\section{OSCHLLATIONS}

Presence of NDR, leaves a possibility that free running oscillations may be generated when the overall resistance in a closed loop becomes less than zero. The loop is usually formed with the stray capacitance which is generally significantly larger than the electrode capacitance. Equations (8) and (9) are able to predict the shape of the oscillations in the low current range. We have however observed one or two transitions with a significant change in the shape of the self sustained oscillations which presumably correspond to transition to the glow regime. The model developed here cannot describe the glow conditions as it assumes almost uniform electric field. In any case, the low current free running oscillations are one of the observables that can be used. For practical purposes it is important to establish ranges of oscillations and stable operation of low current diffuse discharges as a function of the series resistance. Such ranges can be predicted very accurately from the theory $[1,3]$ on the low current end, again the high current limit requires model that includes selfconsistent calculation of the field in the glow regime.

If, however, one wants to obtain reliable data for modeling and consequently obtaining an insight into the relevant processes, it is better to use damped oscillations because they can be made small enough in order not to make large perturbations on the steady state discharge. In addition it is possible to obtain analytic solutions for their properties. In Figure 7. we show an example of experimental data and calculations of the frequency $(\omega)$ and the damping coefficient $(k)$ of such oscillations for hydrogen. Theory gives a very good prediction of the experimental data in most cases. For most gases, however, additional experimental data for secondary electron yields and its dependence on $E / N$ are lacking so fitting of the characteristics of induced oscillations may be a satisfactory source of data needed to predict other properties of the discharge.

It has been shown experimentally [14] that it is possible to damp the oscillations and extend the region of stable operation by increasing the monitoring resistor $R_{\mathrm{m}}$ which is within the loop where oscillations may occur. The condition for stable operation is $R_{\mathrm{m}}>R_{\mathrm{D}}$ and in most cases it is efficient though very high values of $R_{\mathrm{m}}$ are not practical to use. However, as discussed earlier $R_{\mathrm{D}}$ may increase towards the onset of constrictions making it impossible to remove oscillations.

It has been predicted [17] that for mixtures of electronegative and electropositive gases a special type of oscillations will develop in glow and with a frequency much lower than that for the ion feedback driven oscillations that were discussed above. We have observed occurrence of low frequency self sustained oscillations in the glow regime for pure $\mathrm{SF}_{6}$ only at very low $p d$ (see Figure 8.). We could not 
operate the discharge in the exact conditions (10 Torr) used in the theoretical model so it is still uncertain whether the causes for the two low frequency self sustained oscillations are the same.

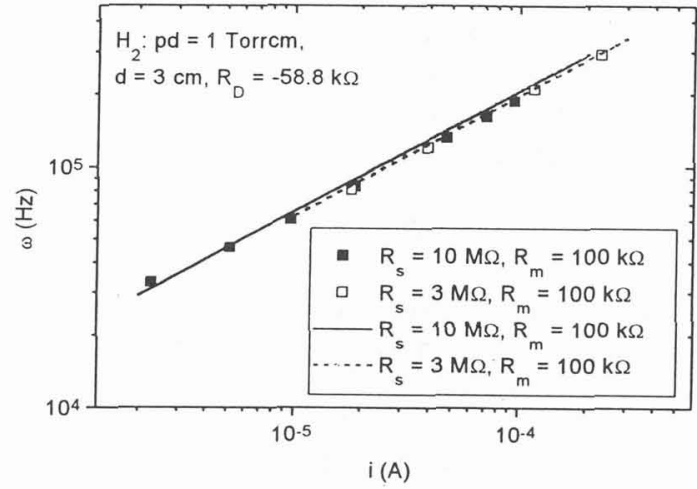

a)

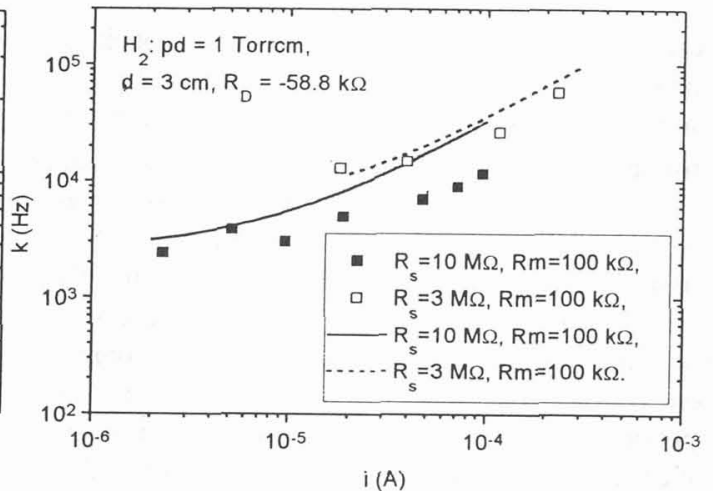

b)

Figure 7: Experimental data and calculations of the a) frequency $(\omega)$ and b) damping coefficient $(k)$ for hydrogen.

\section{CONSTRICTIONS}

Low frequency oscillations are also observed associated with the beginning of constrictions. It was possible to make a pulse of voltage and current into the region close to the onset of constrictions. The pulse stabilizes in the diffuse mode and after some time a transition is made to the constricted discharge. The transition goes through a series of initially-growing low-frequency oscillations. Later, as the mean value of the voltage changes, oscillations are damped. The frequency may be associated with the main process driving the transition. The regime is not identified on the basis of time resolved emission, which is time averaged and the constricted mode dominates. We conclude that the discharge starts in the diffuse mode on the basis of smooth extension from the diffuse conditions. When pulse increases only a little further, the transition is made directly to the constricted discharge.

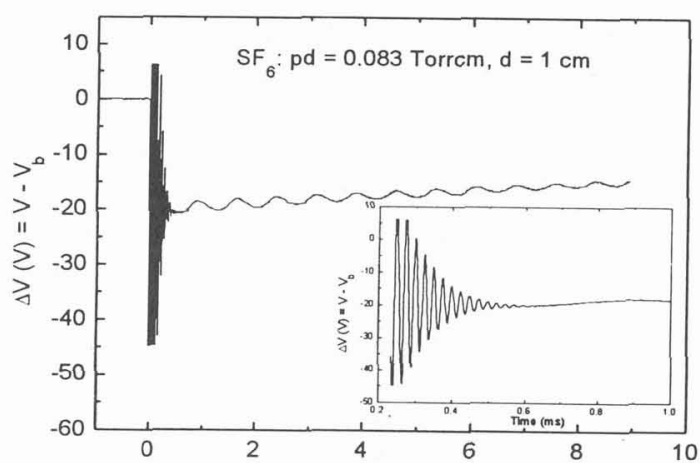

Figure 8: Voltage waveform in $\mathrm{SF}_{6}$ with both the high and low frequency oscillations. The insert shows the first millisecond magnified.

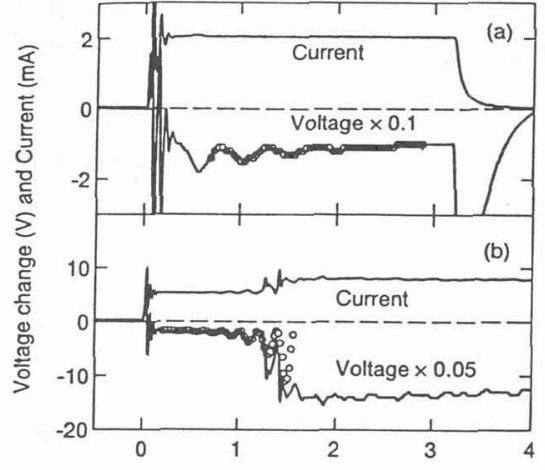

Figure 9: Voltage and current transients in Ar for pressure of 0.12 Torr. The upper trace in each paír shows the discharge current, while the lower trace shows the voltage change. These traces show the transition from a diffuse to constricted regime occurring during the pulse [4].

We have made a systematic study of the onsets of constrictions in a number of gases, hydrogen, argon and nitrogen in particular. Some differences in the behavior of constricted glow discharges are 
observed, as well as the differences due to different $E / N$ or $p d$ values. A particularly interesting situation occurs in argon at 0.12 Torr $\mathrm{cm} \mathrm{[4].} \mathrm{The} \mathrm{discharge} \mathrm{seems} \mathrm{to} \mathrm{concentrate} \mathrm{close} \mathrm{to} \mathrm{the} \mathrm{walls} \mathrm{with} \mathrm{very} \mathrm{small}$ intensity of emission at the axis which is unlike the behavior of other gases and even of the same gas at different $p d$. The observation was labeled as "donut" constriction and it actually proved to be a regular constriction rotating close to the walls with a frequency slightly larger than $1 \mathrm{kHz}$. The reasons for rotation were not fully understood yet.

Comparisons between our observations and some of the available theories indicate that the emission normalized to current remains constant over a wide range of currents [4].

\section{CONCLUSION: WHY AND WHAT NEXT?}

Low current diffuse, or dark Townsend discharges are the simplest form of discharges. Therefore it is essential to achieve a full understanding of their basic physics and to use them to test the models for more complex discharges with strongly inhomogeneous field [18]. For this purpose we need reproducible data obtained under controlled and well defined conditions and we hope that our work may be a step towards providing such data. In particular, such discharges have been used to study low pressure, high $E / N$ situations [10] with a strong non-equilibrium which to a large degree represent the non-equilibrium nonlocal electron transport found in sheaths of dc and if discharges. The most difficult parameter to control is the secondary electron yield from the cathode surface that varies over a large range of values depending on the conditions at the surface. Therefore the only technique that takes care of this problem would be to use the breakdown voltage to establish its value. Even gold plated surfaces have a strongly variable conditions that may be stabilized by running a discharge.

Low current ${ }^{-}$diffuse discharges are also often used to perform swarm experiments [19] and therefore determine data on atomic and molecular processes. Assumption of uniform field that may be essential for such purposes should be verified by following some of the procedures described in this paper. Such experiments are the only method to sufficiently simplify the discharge in order to be able to rule out competing processes and identify some of the physical phenomena such as heavy particle excitation [20], excitation after surface reflection of heavy particles [21] and electron backscattering [10] and its influence on discharge kinetics. Those are just few examples of processes which are usually neglected in discharge modeling but which may be significant under special circumstances and which have been studied in low current discharges i.e. in swarm-like situations.

In addition to our work there is a number of recent theoretical $[16,17 ; 22]$ and experimental [23] studies of low current diffuse discharges which illustrates a continuing interest. Unfortunately we have no room in this paper to give a more detailed review of the entire field.

Finally we may still expect to find and understand some new physics even in such simple systems, and being simple it could even make them well suited for studies of such processes. Constrictions are usually associated with the glow discharge conditions and thus modeled from that viewpoint. Yet the "decision" to change into constriction is "made" in the low current Townsend discharge as a result of processes described here. In particular it is the accuracy and stability of the current where transition takes place that has to be explained on the basis of the low current regime physics, not the glow. Constriction is in fact one of the simplest and first studied forms of selforganization. A simple one dimensional model of competing current channels with the addition of a small perturbation gives the constriction and approximately accurate conditions for the onset of constriction [24]. It is based on the $E N$ dependence of the ionization coefficient only. Of course, it cannot give accurate properties of the discharge once the constriction is achieved but it still describes the basic aspect of selforganization of the charges. Important and still puzzling aspect of the process is the fact that charges do not interact directly under the low current conditions. Interaction exists, as described in this paper but it is indirect, through shielding of the field and with additional nonlinearity brought in by the $E / N$ dependence of the ionization coefficient. 


\section{Acknowledgements}

The authors are grateful to Dr. A.V. Phelps who initiated this program and gave us considerable help and advice in addition to permission to use some of his data. Part of this work was performed during the stay of one of the authors (Z.Lj.P) at Joint Institute for Laboratory Astrophysics, University of Colorado at Boulder. Ministry of Science and Technology of Serbia funded to a small degree part of the project carried out at the Institute of Physics, University of Belgrade. Authors are also grateful to Slobodan Manola and MTT INFIZ for partial support.

\section{References}

[1] Z.Lj. Petrović and A. V. Phelps, Phys. Rev. E 47 (1993) 2806-2815.

[2] B. M. Jelenković, K. Rózsa and A. V. Phelps, Phys. Rev. E 47 (1993) 2816-2824.

[3] A. V. Phelps, Z. Lj. Petrović and B. M. Jelenković, Phys. Rev. E 47 (1993) 2825-2838.

[4] Z.Lj. Petrović and A. V. Phelps, (submitted for publicaton) Phys. Rev. E.

[5] I. Stefanović and Z.Lj. Petrović, Jpn. J. Appl. Phys. 36 (1997) 225-259.

[6] I. Stefanović , Z. Velikić and Z.Lj. Petrović, in Contributed Papers of the $22^{\text {nd }}$ International Conference on Phenomena in Ionized Gases, Hoboken, New Jersey,USA (1995) pp. 123-124.

[7] R. S. Sigmond, in Proceedings of the $4^{\text {th }}$ International Conference on Ionization Phenomena in Gases, Uppsala (1959), ed. N. R. Nilsson (North-Holland, Amsterdam, 1960) pp. 189-194.

[8] M. J. Druyvesteyn and F. M. Penning, Rev. Mod. Phys. 12 (1940) 87-174.

[9] M. Hayashi and Y. Miyoshi, Bulletin Nagoya Inst. Technology 13 (1962) 339-345.

[10] V. Stojanović and Z.Lj. Petrović, (to be published).

[11] A. V. Phelps, (unpublished).

[12] V. N. Melekhin and N. Yu. Naumov, Sov. Phys. Tech. Phys. 29 (1984) 888-892.

[13] V. N. Melekhin, N. Yu. Naumov and N. P. Tkachenko, Sov. Phys. Tech. Phys 32 (1987) 274-279.

[14] A. V. Phelps, JILA Data Center Report. 35 (1993)

[15] S.B.Vrhovac, I. Stefanović and Z.Lj.Petrović, Proc. XXIII ICPIG (Toulouse) II (1997) 44-45.

[16] Z.Lj. Petrović and A. V. Phelps, (unpublished).

[17] I. Pérès and L. C. Pitchford, J. Appl. Phys. 78 (1995) 774-782.

[18] V. I. Kolobov and A. Fiala, Phys. Rev. E 50 (1994) 3018-3032.

[19] Z.M. Jelenak, Z.B. Velikić, J.V. Božin, Z.Lj. Petrović and B.M. Jelenković, Phys Rev. E 47 (1993) 3566-35.73.

[20] V.D. Stojanović, B.M. Jelenković and Z.Lj. Petrović, J. Appl. Phys. 81 (1997) 1601-1603.

[21] Z.Lj.Petrović, B.M. Jelenković and A.V.Phelps; Phys. Rev. Lett. 68 (1992) 325-327.

[22] I.D.Kaganovich, M.A.Fedotov and L.D.Tsendin, Tech. Phys. 39 (1994) 241-245.

[23] K.Rozsa, L.Szalai and Z.Donko, Europhys. Abstracts 20 E (1996) 161-162.

[24] Z.Lj. Petrović, (unpublished). 